\title{
Surface models and true-color sectioned images of hypothalamic nuclei and its neighboring structures
}

\author{
Yaqian You ${ }^{\mathrm{a}, \mathrm{d}}$, Chung Yoh Kim ${ }^{\mathrm{b}}$, Gen Yan ${ }^{\mathrm{a}, \mathrm{c}, *}$ and Jin Seo Park ${ }^{\mathrm{b}, *}$ \\ ${ }^{a}$ Department of Radiology, Affiliated Hospital of Jiangnan University, Wuxi, Jiangsu, China \\ ${ }^{\mathrm{b}}$ Department of Anatomy, Dongguk University School of Medicine, Gyeongju, Korea \\ ${ }^{\mathrm{c}}$ Department of Radiology, Second Affiliated Hospital of Xiamen Medical College, Xiamen, Fujian, China \\ ${ }^{\mathrm{d}}$ Department of Anatomy, Wuxi School of Medicine, Jiangnan University, Wuxi, Jiangsu, China
}

\begin{abstract}
.
BACKGROUND: Knowledge regarding the hypothalamic nuclei is essential for understanding neuroanatomy and has substantial clinical relevance.

OBJECTIVE: The aim was to contribute to elucidate the complex hypothalamic architecture for research and provide an anatomical basis for clinical brain operation.

METHODS: In this research, high-resolution and true-color sectioned images from Visible Korean were employed for hypothalamic nuclei and neighboring structures surface modeling, and a high-resolution three-dimensional atlas of the hypothalamus was created.

RESULTS: Surface models of 26 structures including the hypothalamic nuclei and its neighboring structures were produced, which contained 5 anterior hypothalamic areas' nuclei, 4 intermediate hypothalamic areas' nuclei, 3 lateral hypothalamic areas' nuclei, and 2 posterior hypothalamic areas' nuclei, as well as 12 hypothalamic neighboring structures.

CONCLUSIONS: The study evaluated the topographical anatomy of the hypothalamic nuclei and its neighboring structures based on true-color and highresolution sectioned images of Visible Korean.
\end{abstract}

Keywords: Hypothalamus, Visible Korean, true-color, sectioned image, three-dimensional model

\section{Introduction}

The hypothalamus is a small region of the diencephalon located below the thalamus, on the bilateral walls and floor of the third ventricle. It consists of several nuclei in four areas (anterior-lateralintermediate-posterior), of which the rostral part exerts prominent regulation in predictive homeostasis, as opposed to the caudal parts that are primarily involved in reactive homeostasis [1,2]. Bettering understanding of the hypothalamus is beneficial in diagnosing various relevant diseases that require detailed anatomic analyses of the hypothalamus. For detailed analyses, three observation methods are employed as follows: the first is the histological staining of the nervous tissues in brain section slides [3,4]; however, in

\footnotetext{
${ }^{*}$ Corresponding authors: Gen Yan, Department of Radiology, Second Affiliated Hospital of Xiamen Medical College, Xiamen, Fujian 361021, China. Tel.: +86 1881620 5526; E-mail: gyan@stu.edu.cn. Jin Seo Park, Department of Anatomy, Dongguk University School of Medicine, Gyeongju 38066, Korea. Tel.: +82 54770 2402; E-mail: park93@ dongguk.ac.kr.
} 
Table 1

Twenty-six structures selected for making surface models and 27 structures selected for making annotated sectioned images

\begin{tabular}{|c|c|c|}
\hline System & & Structures \\
\hline Skeletal & \multicolumn{2}{|l|}{ Crista galli* } \\
\hline Endocrine & \multicolumn{2}{|c|}{ Pituitary gland (adenohypophysis, neurohypophysis), infundibulum } \\
\hline Vascular & \multicolumn{2}{|c|}{ Anterior cerebral artery*, pericallosal artery*, inferior sagittal sinus* } \\
\hline \multirow[t]{2}{*}{ Nervous } & Hypothalamus & $\begin{array}{l}\text { Anterior hypothalamic area (anterior nucleus of hypothalamus, preoptic nucleus, } \\
\text { paraventricular nucleus, suprachiasmatic nucleus, supraoptic nucleus), Intermediate } \\
\text { hypothalamic area (dorsomedial nucleus, arcuate nucleus, periventricular nucleus, } \\
\text { ventromedial nucleus of hypothalamus), Lateral hypothalamic area (lateral preoptic area, } \\
\text { lateral tuberal nuclei, tuberomammillary nucleus), Posterior hypothalamic area } \\
\text { (mammillary body, posterior nucleus of hypothalamus) }\end{array}$ \\
\hline & $\begin{array}{l}\text { Structures other } \\
\text { than hypothalamus }\end{array}$ & 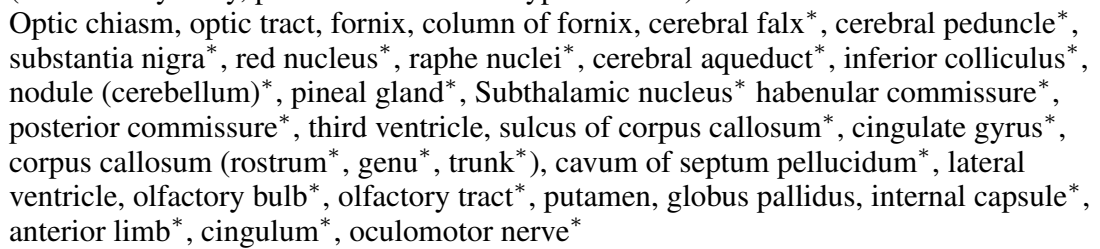 \\
\hline
\end{tabular}

${ }^{*}$ Annotated structures.

stained slides, only gray and white matter are observed and the actual color of the brain is unidentifiable. The second is the use of computed tomographs (CTs) and magnetic resonance images (MRIs), which are in almost all current studies and clinics [5-7]. However, using radiation and magnetic resonance to target the hypothalamus and, in particular, specific nuclei and white bundles are very vague approaches and does not provide useful information because the hypothalamus is small compared to the other brain structures and is located deep in the brain. The third is the observation of three-dimensional (3D) models of the hypothalamus. Some 3D models of the hypothalamus have been obtained using CTs and MRIs [5], but they are vague and incomprehensible because of low resolution and the gray scale of CTs and MRIs.

The hypothalamus is considered to be a link structure between the nervous and the endocrine system, its main function being to maintain the homeostasis of the body, therefore, around the hypothalamus, there are many structures connected to it by both nervous and chemical pathways, important structures adjacent to the hypothalamus include pituitary gland, optic chiasma, globus pallidus, putamen, thalamus, and so on [8].

In order to solve this problem, high resolution and real-color sectioned images are necessary to fully explore the hypothalamus. The purpose of this study was to provide a 3D atlas of the hypothalamus based on surface models and sectioned images, to learn sectional neuroanatomy with the hope to broaden our understanding of the complex hypothalamic architecture.

\section{Materials and methods}

Sequence sectioned images (intervals, $0.1 \mathrm{~mm}$; pixel size, $0.1 \mathrm{~mm} \times 0.1 \mathrm{~mm}$; color depth, 48 bits color) of a cadaver head of the Visible Korean [9], were used for this study. From the sectioned images, we selected the images of the coronal plane (intervals, $0.1 \mathrm{~mm}$ ); subsequently, we picked one out of every 10 images (intervals, $1 \mathrm{~mm}$ ) for segmentation and annotation.

Twenty-six structures, hypothalamic nuclei and its neighboring structures, were selected for segmentation and 27 structures were selected for annotations on the sectioned images (Table 1).

On the coronal plane of sectioned images, the locations of the hypothalamic nuclei and tracts were identified based on some anatomical landmarks, such as the third ventricle and optic chiasma. In these 


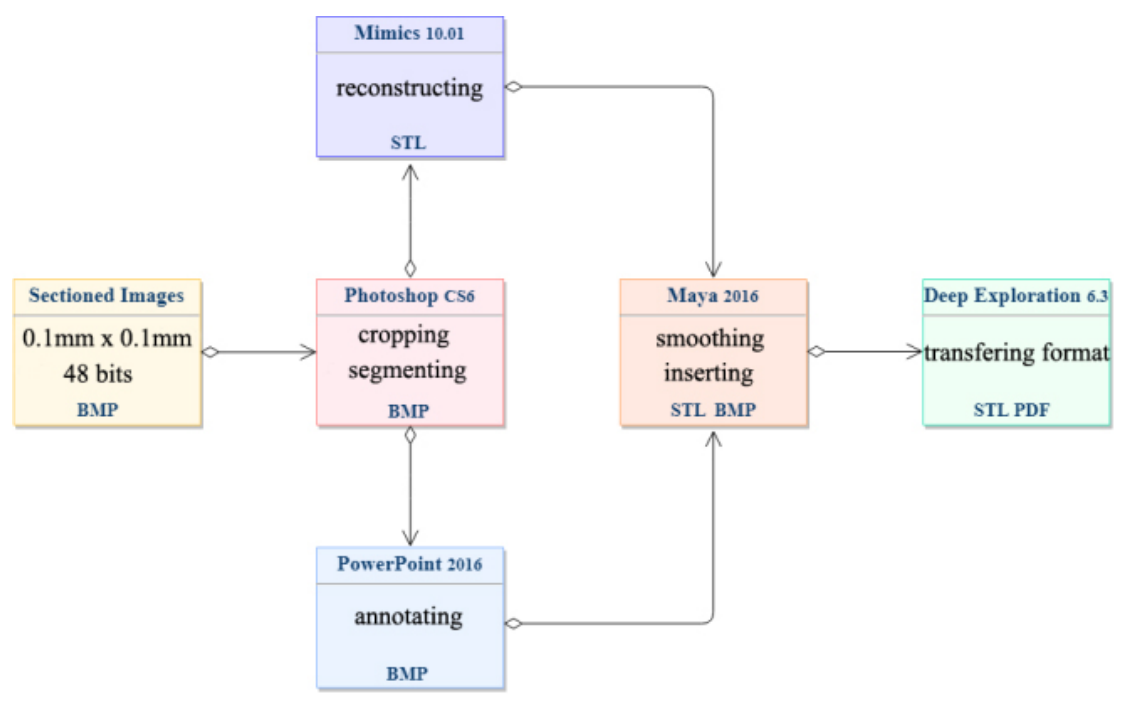

Fig. 1. Flow-chart showing the process of this experiment.

sectioned images, the boundaries of the structures were outlined semi-automatically with the Lasso tool or the Magnetic Lasso tool and saved as new layers in Adobe Photoshop CS6 (Adobe Systems, Inc., San Jose, CA, USA) [10,11]. The outlines of the segmented structures were then extracted and filled with colors automatically using Action and were saved in BMP format with Photoshop to create the segmented images (interval, $1.0 \mathrm{~mm}$; pixel size, $1.0 \times 1.0 \mathrm{~mm}$; color depth, 24 bits color).

Surface models were reconstructed automatically (Fig. 4). The models were exported as stereolithography (STL) files, which were then imported to Maya 2016 (Autodesk Inc., San Rafael, CA, USA) for smoothing followed by embedding with the annotated coronal sectioned images using that software (Fig. 4) [12].

On the coronal plane of sectioned images, we first identified the surrounding structures and then annotated their names using PowerPoint 2016 (Microsoft Corp., Seattle, WA, USA) (Table 1), then saving as BMP format files. Finally, these BMP files were imported to the software of Maya for resizing, so as to fit the STL structure files.

After importing the STL files to Maya, a polygonal plane on the grid was created, and the coronal sectioned image was assigned to the polygonal plane. Its scale and spatial position were adjusted to match the STL structures, and subsequently, all the annotated coronal sectioned images ( 87 pieces) were inserted in the same way (Fig. 4). These sectioned images were also saved as an STL format in Maya. Finally, we assembled these aligned sectioned images with surface models in Deep Exploration 6.3 (Right Hemisphere, Fremont, CA, USA) (Fig. 5) and renamed these files. We then exported them to a Portable Document Format (PDF) file.

The process of 3D reconstruction and annotation were showed in flow-chart (Fig. 1). All of the image data are based on Visible Korean, we didn't do any other statistical analysis for the image processing and $3 \mathrm{D}$ reconstruction.

\section{Results}

The hypothalamus nuclei from rostral to caudal, a series of coronal sectioned images contained hypothalamus were selected (Fig. 2). Nesting the 3D reconstruction models into the sectioned images, 

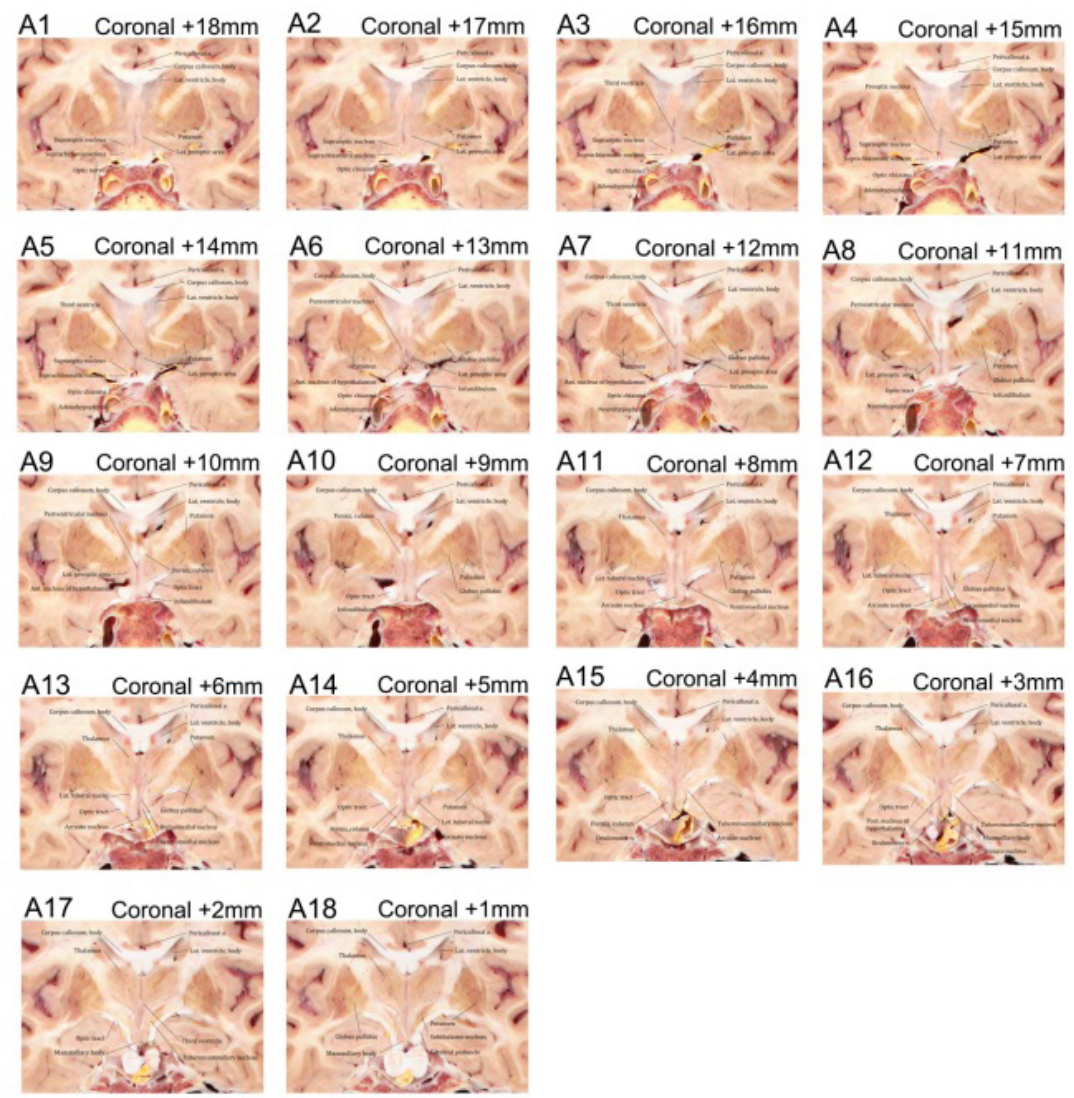

Fig. 2. Serial coronal plane sectioned images contained hypothalamic (A1-A18). The coordinate for each slice has been indicated (Coronal $+18 \mathrm{~mm}-$ Coronal $+1 \mathrm{~mm})$.

the hypothalamus nuclei were accurately discernible in the Visible Korean (Fig. 3). In addition, we can observe the annotated structures in high-resolution images clearly as in atlas.

Surface models of 26 structures including the hypothalamic nuclei and its neighboring structures were produced in STL files (Total file size, 20.1 MB). All the STL files were assembled in a PDF file (File size, 94.3 MB). In the PDF file, all surface models were displayed together, with free rotation scaling, so as to provide a clear and intuitive understanding of the hypothalamus. In the PDF file, we identified the accurate position and shape of the structures by combining the surface models with the 27 annotated structures on the coronal plane of sectioned images (Fig. 4).

The hypothalamic nuclei are distributed on both sides of the third ventricle symmetrically. According to their position, these nuclei can be divided into four neural zones: the anterior hypothalamic area, the intermediate hypothalamic area, the lateral hypothalamic area, and the posterior hypothalamic area (Fig. 5A and B and Table 1). In the surface models with the sectioned images, the location and shape of the hypothalamic nuclei and the neighborhood structures could be identified as follows.

First, the anterior hypothalamic area contained the largest number of nuclei. They lay in the frontal side of the third ventricle and the hypothalamus; therefore, this nuclei group was named the anterior nucleus of the hypothalamus that contains the anterior nucleus of the hypothalamus, the preoptic nucleus, paraventricular nucleus, suprachiasmatic nucleus, and preoptic nucleus (Fig. 5C and Table 1). Among these nuclei, the suprachiasmatic nucleus and the supraoptic nucleus are located above the optic chiasma 

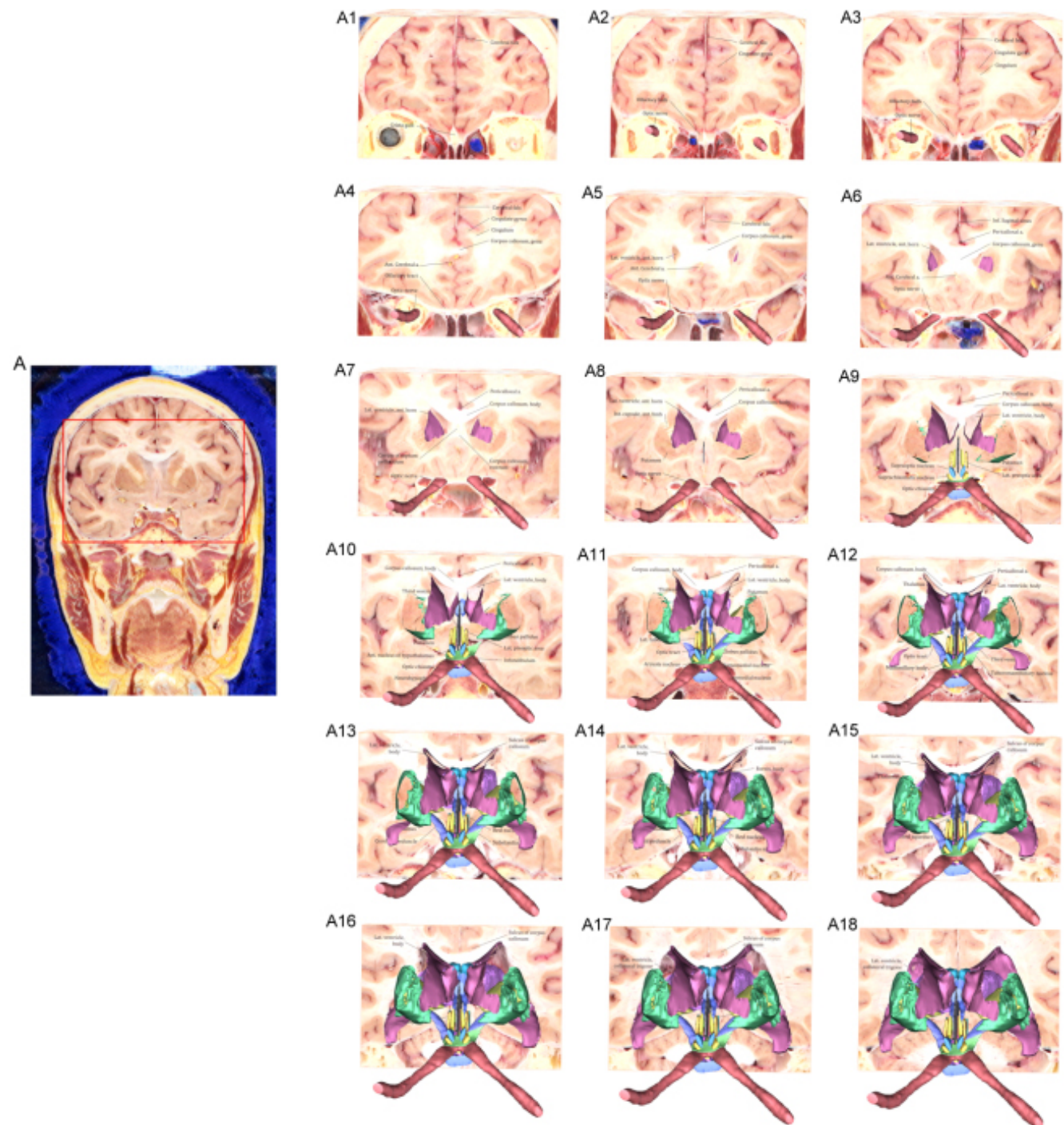

Fig. 3. Serial coronal plane sectioned images showing contained hypothalamus and its neighboring structures that from rostral to caudal. Put one every five sheets (A1-A18), all sectioned images were cropped from the Visible Korean using Photoshop (A).

but lie beneath the preoptic nucleus; behind the preoptic nucleus is the paraventricular nucleus, and the anterior nucleus of the hypothalamus is below it.

Second, the intermediate hypothalamic area contained four nuclei (dorsomedial nucleus, ventromedial nucleus, arcuate nucleus, and periventricular nucleus). Because these nuclei are located in the middle of the hypothalamus and close to the third ventricle, we called it the intermediate hypothalamic area. Among these, the periventricular nucleus is closely attached to the third ventricle tightly, and the dorsomedial and ventromedial nuclei lie slightly outside of that. Arcuate nucleus, the only asymmetric mononuclear in the hypothalamus, is located at the base of the third ventricle (Fig. 5D and Table 1).

Third, the lateral hypothalamic area located in the bilateral temporal side of the hypothalamus and lateral side of other nuclei consisted of the lateral preoptic area, lateral tuberal nuclei, and tuberomammillary nucleus. The three nuclei line up along the hypothalamus from the front to the back (Fig. 5E and Table 1). 


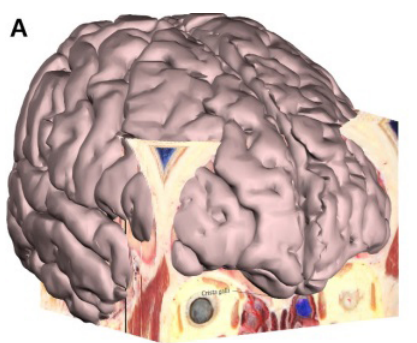

B
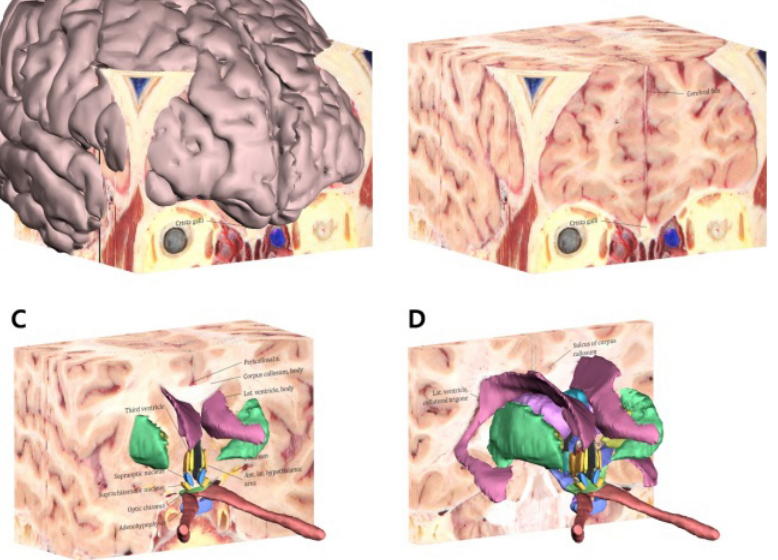

D

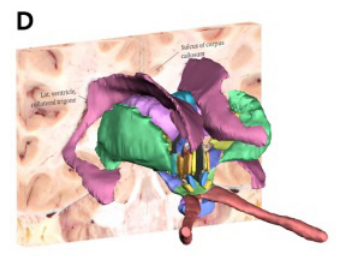

Fig. 4. Surface models with the sectioned images in the PDF file. The surface model of the whole cerebrum with all the coronal sectioned images (A) and only the coronal sectioned images (B) can be displayed using Adobe Reader. After peeling some sectioned images, the hypothalamus and its neighboring structures appear on the rest of the sectioned images $(C, D)$.

Fourth, the posterior hypothalamic area contained the mammillary body and the posterior nucleus of the hypothalamus in the posterior side of the hypothalamus. They were symmetrically distributed on both sides of the third ventricle (Fig. 5F and Table 1).

In addition, structures adjacent to the hypothalamus have also been reconstructed, such as the thalamus, pituitary gland, third ventricle, infundibulum, optic chiasma, optic tract, optic nerve, and fornix (Fig. 4 and Table 1). The structures surrounding the hypothalamus were numerous on account of being located deep in the brain. Determining the presence of the accompanying structures and even the outline of these structures is a vital factor in locating the hypothalamic nucleus and reconstructing it. Besides, we put the hypothalamic nuclei and its neighboring structures in a spatial system; its co-existence with adjacent structures helped us grasp its distribution as a whole and have a clearer understanding of its positional relationship, which clarified our understanding of the complicated geographical location of the hypothalamus (Figs 4-6).

\section{Discussion}

The hypothalamus, a site of integration for the endocrine, autonomic, and behavioral responses, is that portion of the diencephalon in humans that lies inferior to the thalamus [1]. It is difficult to learn the anatomy of the hypothalamus and its neighboring structures because it is difficult to identify the hypothalamus and the neighboring structures using existing methods, due to their limitations. To remedy this problem, we used high resolution and true-color sectioned images and reconstructed the surface models based on these sectioned images.

In contrast to histological stained slides [3,4] our sectioned images with high-resolution and true-color are optimal for observing the hypothalamic nuclei with minute volume, large quantity, and similar colors (Figs 4 and 5).

In CTs and MRIs, the appearances of the nuclei and white bundles of the hypothalamus were very vague and illegible [5-7]. For the purpose of segmenting based on color, density, and position, high resolution and real color of the sectioned images were more suitable. The true-color and size allow us 
A
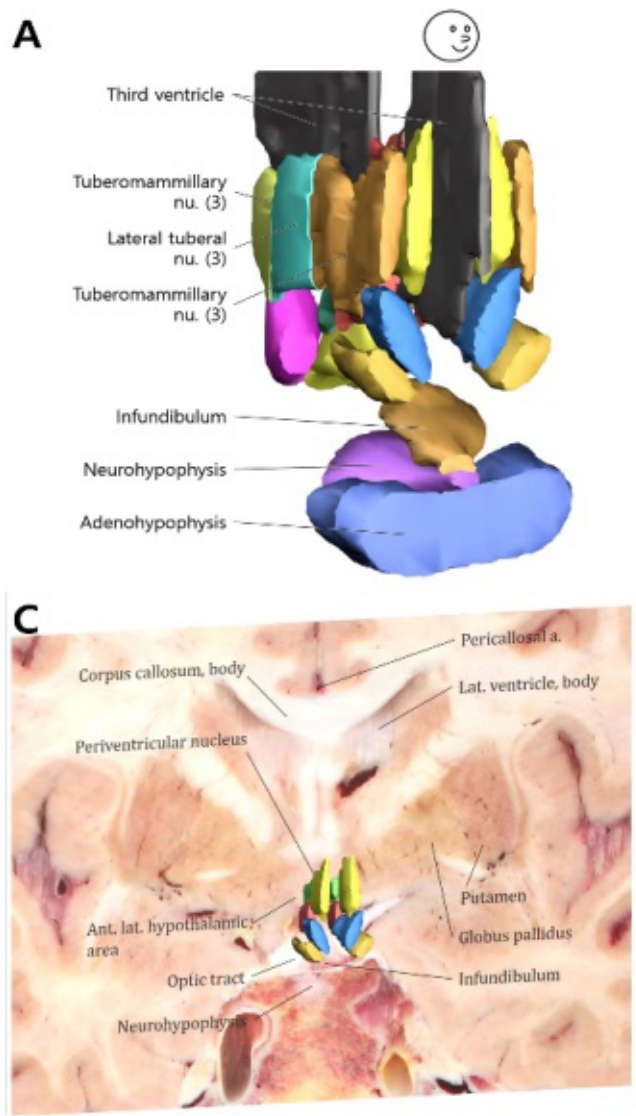

$\mathbf{E}$

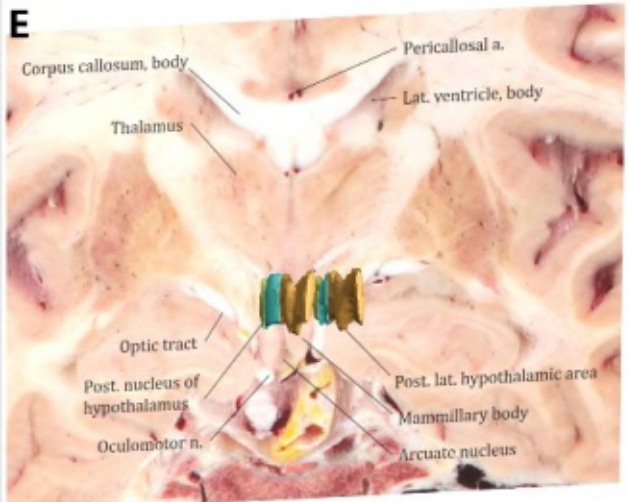

B
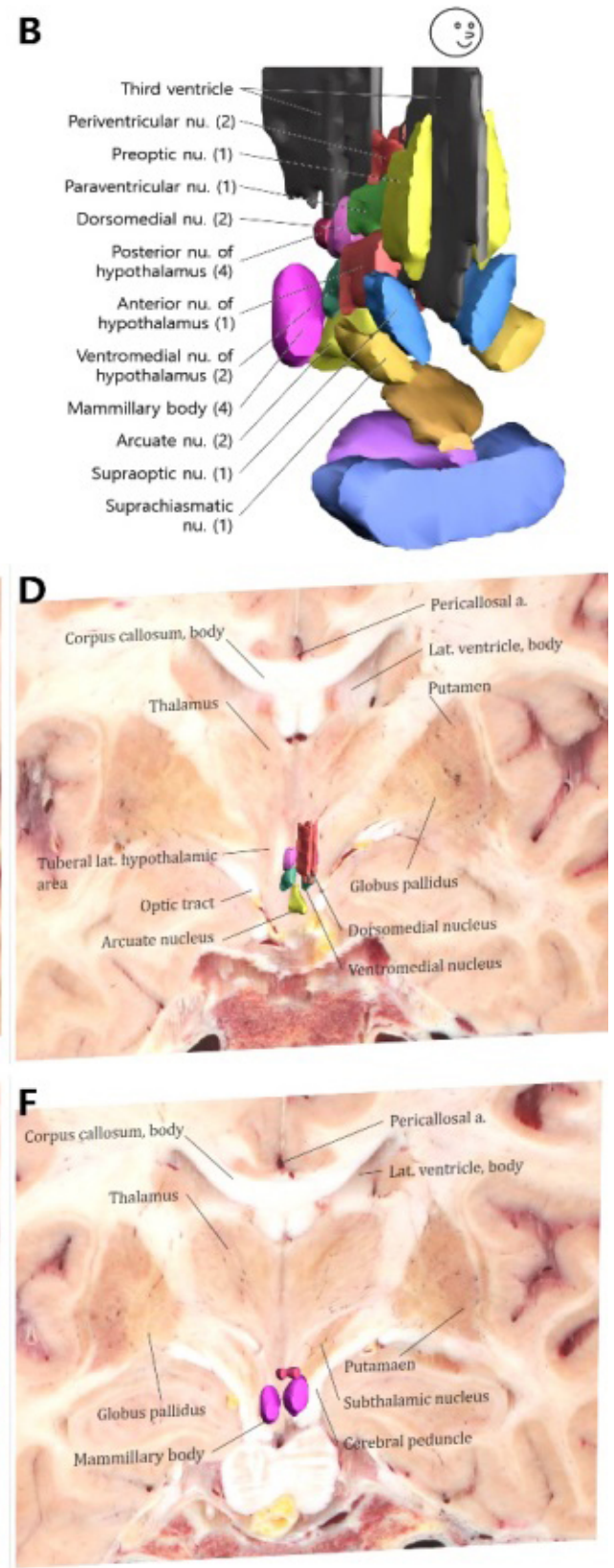

Fig. 5. Surface models and sectioned images of the four neural zones of the hypothalamus. In only the surface models, the hypothalamic nuclei are shown with the anterior (1), intermediate (2), lateral (3), and posterior (4) hypothalamic areas (A) and without the lateral hypothalamic area (B). On the coronal plane of sectioned images with surface models, 5 nuclei in the anterior hypothalamic area (C), 4 nuclei in the intermediate hypothalamic area (D), 3 nuclei in the lateral hypothalamic area (E), and 2 nuclei in the posterior hypothalamic area (F) can be identified stereoscopically.

to distinguish each structure of the diencephalon and even the hypothalamus. Such a well-preserved structure with high-resolution can solve the problem of ambiguity (Figs 3 and 4).

Usually, the 3D models of the hypothalamus were made from MRIs and CTs. However, they were very 

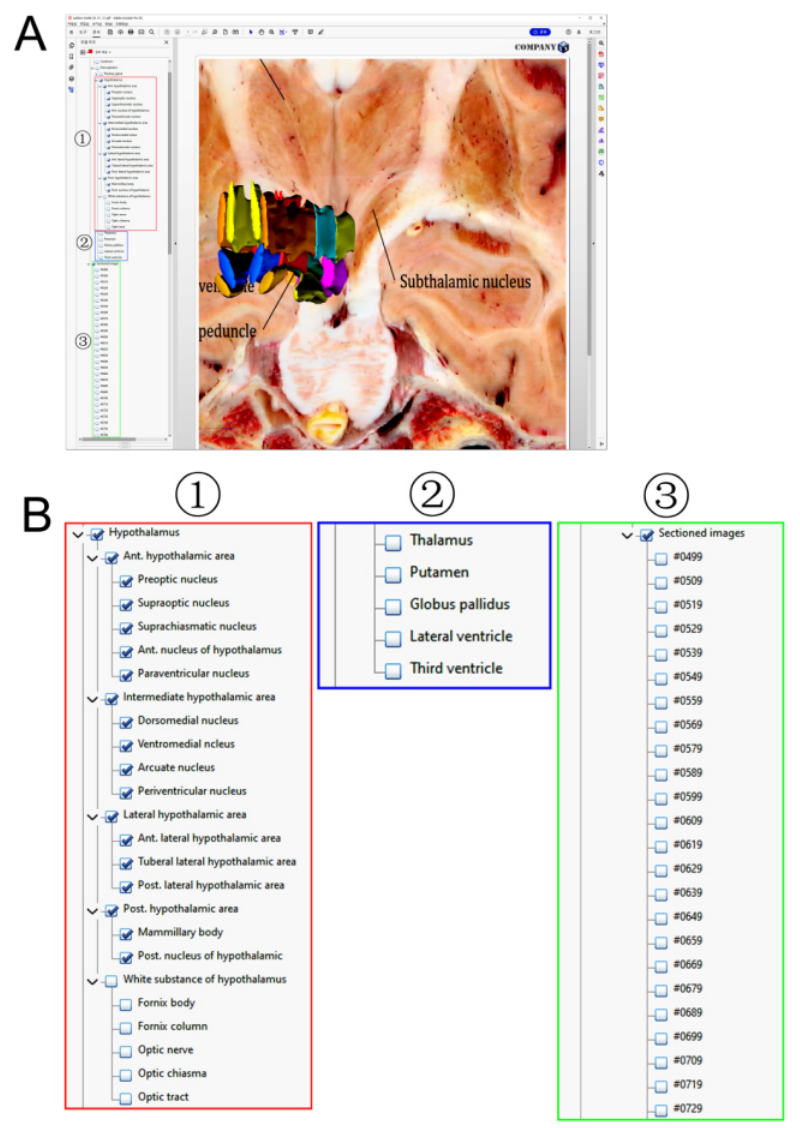

Fig. 6. Surface models with the sectioned images and model tree. Detailed structures in the deep brain can be displayed, and affiliations of all structures can be displayed in the model tree (A), zoomed view of the model tree were displayed (B), with hypothalamus (1), neighboring structures (2) and sectioned images (3).

vague [13], because of low resolution and the gray scale of MRIs and CTs. In our study, we have created 3D models based on high-resolution sectioned images that retain the true color, shape, and position. The sectioned images guarantee the authenticity and accuracy of the 3D model to the greatest extent (Figs 3-5).

In this study, we selected high-resolution sectioned images of the coronal plane for segmentation and selected the STL and PDF formats for storage and distribution for the following reasons. First, we selected the sectioned images for segmentation from the Visible Korean [9]. The most crucial and difficult process was affirming and segmenting the structures on 2D raw images in creating computer assisted 3D reconstruction. In this study, high-resolution sectioned images were chosen, which are helpful for clearly identifying the complex distributions of the hypothalamic nuclei and its related structures, especially for rendering the structures' boundaries (Figs 4 and 5).

Second, we selected the coronal plane for segmentation. Even though the sectioned images have three directions, namely horizontal, sagittal, and coronal planes [9], we finally chose the coronal plane for segmenting. The reason for this was that the locations of almost all of the hypothalamic nuclei were bilateral axisymmetric based on the third ventricle. The selected coronal plane for labeling and reconstructing the nuclei may facilitate researchers' understanding of their location and distribution (Figs 3 and 4). 
Third, we selected STL and PDF formats for storage and distribution. The STL format is used very widely in surface models because the STL format can be opened in any 3D software, even Microsoft Windows 10 default app (Paint 3DTM). Therefore, users can easily identify the surface models. With the PDF format, the observers are able to browse through surface models easily and swiftly with a common application software such as Adobe Acrobat system [14].

Recently, many studies testing that deep brain stimulation as a treatment in advanced nervous system disease, such as Alzheimer's disease and Parkinson's disease $[15,16]$. When doing surgery of deep brain stimulation, subthalamic nucleus, thalamus and globus pallidus are the brain areas for placing electrodes [17]. From an anatomy point of view, clearing the location of the surrounding structures is meaningful for clinical operations.

However, although we used high-resolution images to reconstructed hypothalamus nuclei, the surface models still wear pseudo-color, we will focus on solving this problem in the future research. And, this study was a preliminary work and consequently there is plenty room to improve this methodology, for example, improving rendering, automatizing many of the tasks, designing an own graphic interface.

By combing surface models of the hypothalamus and the annotated sectioned images of the deep brain, users can have a comprehensive understanding of the $3 \mathrm{D}$ overall spatial state and the $2 \mathrm{D}$ primitive existence position of the hypothalamus in detail (Figs 5 and 6). Besides, these studies are expected to provide an anatomical basis for clinical operation. The PDF file of this study is distributed freely at the author's homepage (http://neuroanatomy.kr).

\section{Acknowledgments}

This study was supported by the Science and Technology Program of Fujian Province of China (No. 2017D017); The Joint Funds for the Health and Education of Fujian Province, China (No. 2019-WJ-31); The Institute of Respiratory Diseases, Xiamen Medical College (No. HXJB-06, 15); and The Overseas Research Program of Jiangnan University.

\section{Author contributions}

Yaqian You designed the experiments and wrote the manuscript; Chung Yoh Kim performed the experiments; Gen Yan and Jin Seo Park designed the experiments and wrote the manuscript.

\section{Conflict of interest}

The authors declare no potential conflict of interest.

\section{References}

[1] Toni R, Malaguti A, Benfenati FMartini L. The human hypothalamus: A morpho-functional perspective. J Endocrinol Invest. 2004; 27: 73-94.

[2] Lechan RMToni R, Functional Anatomy of the Hypothalamus and Pituitary, in: Endotext, Feingold KR, Anawalt B, Boyce A, Chrousos G, de Herder WW, Dhatariya K, Dungan K, Grossman A, Hershman JM, Hofland J, Kalra S, Kaltsas G, Koch C, Kopp P, Korbonits M, Kovacs CS, Kuohung W, Laferrère B, McGee EA, McLachlan R, Morley JE, New M, Purnell J, Sahay R, Singer F, Stratakis CA, Trence DL, Wilson DP, eds., MDText.com, Inc. Copyright (C)2000-2021, MDText.com, Inc., South Dartmouth (MA), 2000. 
[3] Braak H, Braak E. Anatomy of the human hypothalamus (chiasmatic and tuberal region). Prog Brain Res. 1992; 93: 3-14; discussion 14-16. doi: 10.1016/s0079-6123(08)64559-8.

[4] Axer H, Berks G, Keyserlingk DG. Visualization of nerve fiber orientation in gross histological sections of the human brain. Microsc Res Tech. 2000; 51: 481-492. doi: 10.1002/1097-0029(20001201)51:5<481::Aid-jemt11>3.0.Co;2-n.

[5] Baroncini M, Jissendi P, Balland E, Besson P, Pruvo JP, Francke JP, Dewailly D, Blond S, Prevot V. MRI atlas of the human hypothalamus. Neuroimage. 2012; 59: 168-180. doi: 10.1016/j.neuroimage.2011.07.013.

[6] Lemaire JJ, Nezzar H, Sakka L, Boirie Y, Fontaine D, Coste A, Coll G, Sontheimer A, Sarret C, Gabrillargues J, De Salles A. Maps of the adult human hypothalamus. Surg Neurol Int. 2013; 4: S156-163. doi: 10.4103/2152-7806.110667.

[7] Sienkiewicz T, Sergiel A, Huber D, Maślak R, Wrzosek M, Podgórski P, Reljić S, Paśko Ł. The Brain Anatomy of the Brown Bear (Carnivora, Ursus arctos L., 1758) Compared to That of Other Carnivorans: A Cross-Sectional Study Using MRI. Front Neuroanat. 2019; 13: 79. doi: 10.3389/fnana.2019.00079.

[8] Gabriela Pop M, Crivii C, Opincariu I. Anatomy and Function of the Hypothalamus, in: Hypothalamus in Health and Diseases, 2018.

[9] Park JS, Chung MS, Shin DS, Har D, Cho Z, Kim Y, Han J, Chi J. Sectioned Images of the Cadaver Head Including the Brain and Correspondences With Ultrahigh Field 7.0 T MRIs. Proceedings of the IEEE. 2009; 97: 1988-1996. doi: 10.1109/JPROC.2009.2025524.

[10] Park JS, Chung MS, Hwang SB, Lee YS, Har DH. Technical report on semiautomatic segmentation using the Adobe Photoshop. J Digit Imaging. 2005; 18: 333-343. doi: 10.1007/s10278-005-6704-1.

[11] Park JS, ed. Cross-sectional atlas of the human head: With 0.1-mm pixel size color images. Springer Publishers, Singapore; 2017.

[12] Chung B, Park JS. Whole Course of Pallidothalamic Tracts Identified on the Sectioned Images and Surface Models. Clinical Anatomy. 2019; 33. doi: 10.1002/ca.23468.

[13] Lambert C, Zrinzo L, Nagy Z, Lutti A, Hariz M, Foltynie T, Draganski B, Ashburner J, Frackowiak R. Confirmation of functional zones within the human subthalamic nucleus: Patterns of connectivity and sub-parcellation using diffusion weighted imaging. Neuroimage. 2012; 60: 83-94. doi: 10.1016/j.neuroimage.2011.11.082.

[14] Shin DS, Jang HG, Park JS, Park HS, Lee S, Chung MS. Accessible and informative sectioned images and surface models of a cadaver head. J Craniofac Surg. 2012; 23: 1176-1180. doi: 10.1097/SCS.0b013e31825657d8.

[15] Hamani C, Florence G, Heinsen H, Plantinga BR, Temel Y, Uludag K, Alho E, Teixeira MJ, Amaro E, Fonoff ET. Subthalamic Nucleus Deep Brain Stimulation: Basic Concepts and Novel Perspectives. eNeuro. 2017; 4. doi: 10.1523/eneuro.014017.2017.

[16] Mao Z-q, Yu X, Ling Z, Jia J, Pan L, Xu X, Cui Z-q, Han Y, Wang S-s, Liang S-1. Deep brain stimulation for treatment of severe Alzheimer's disease: Study protocol for a prospective, self-controlled, phase I trial (case observation). Asia Pacific Journal of Clinical Trials: Nervous System Diseases. 2017; 2: 66-71.

[17] Deep brain stimulation (DBS) [homepage on the Internet]. Mayfield Brain\&Spine. Available from: https://mayfieldclinic. com/pe-dbs.htm. 